\title{
From Concept to Reality to Vision *
}

\author{
Frank Wilczek ${ }^{\dagger}$
}

June 15, 2018

\begin{abstract}
I take a brief look at three frontiers of high-energy physics, illustrating how important parts of our current thinking evolved from earlier explorations at preceding frontiers.
\end{abstract}

Elucidating the basic nature of the strong interaction was a vast enterprise to which many gifted scientists devoted their best efforts and made wonderful contributions. While the subject is far from finished - the dramatic developments I'll be discussing this afternoon [1] bring that home! - I think it is clear that the foundations are secure. QCD, as the basic theory, is here to stay. It is a marvelous theory, which cleanly embodies mathematical ideas of great depth and beauty. Above all QCD demonstrates, in a unique way, the power of relativistic quantum field theory to produce an amazing wealth of phenomena (asymptotic freedom, jets, confinement, mass generation, resonance spectroscopy, chiral symmetry breaking, anomaly dynamics, ...) in harmony with the observed facts of Nature.

David Gross has just described for you the whirlwind of events and discoveries that led us to propose this theory for the strong interaction, reinforced with concrete reasons to believe in it (and no other!), and packaged with proposals for critical, quantitative experimental tests. I don't want to repeat the details, but only want to endorse what David has already emphasized, that he and I were fortunate indeed to be in a position to leverage a vast accumulation of knowledge and technique built up by a big international community of scientists over decades of dedicated work, much of it frustrating and not properly recognized. As members of this community we should all be proud of our joint achievement.

I'll freely admit that back in 1973 I didn't begin to anticipate the progress in experiment and theory that would bring our subject to the level where it is today. I had some hope that deep inelastic scattering experiments and perhaps measurements of electron-positron annihilation (the total cross section) would be made more precise, maybe precise enough that with careful analysis one would see hints of scaling deviations in the form we predicted, and thereby gradually build up a case for the correctness of QCD. Of course, reality has far outrun these expectations. One of the great joys of my life in physics has been to participate in the process - something like parenthood - whereby unshaped concepts mature in surprising ways into concrete realities, which then engender new visions. I'd like briefly to share with you three examples, in each case mixing a little nostalgia with pointers to the future.

\footnotetext{
${ }^{*}$ Speech in acceptance of European Physical Society prize for high energy physics, Aachen, August 2003.

${ }^{\dagger}$ wilczek@mit.edu
} 


\section{From Running Coupling to Quantitative Unification to Supersymmetry}

Running of gauge theory couplings, and in particular the peculiar anti-screening behavior we call asymptotic freedom, was first established by straight unguided calculation 2, 3. It was first applied to renormalization group equations for deep Euclidean Green's functions and Wilson coefficients in operator product expansions, enabled through a rather cumbersome formalism to describe a very few physical processes [4, 5, 6]. Before long antiscreening was understood in terms accessible to intuition [7]. And by now of course we've learned to exploit the concept much more boldly and confidently, and with great success. Quark and gluon degrees of freedom are identified directly in the energy-momentum flow of jets, and their basic couplings are made manifest in the iconic three-jet processes seen at LEP. Comparing the frequency of such events, at different energies, exhibits the running in as clear and elementary a form as one could ask for.

The calculation of running, of course, extends immediately to electroweak interactions. (Indeed, my original interest in it largely arose from this angle.) It was put to brilliant use in the famous work of Georgi, Quinn, and Weinberg [8], who indicated through its use dreams about unification of interactions (Pati and Salam [9, Georgi and Glashow [10]) could be brought down to earth. One could check concretely whether the observed, unequal couplings might result from running a single coupling from ultra-short to accessible distances. A few years later Dimopoulos, Raby, and I realized [11] (to my great surprise, initially) that including the effects of low-energy supersymmetry, which is quite a drastic expansion of the physics, makes only comparatively small changes in the predictions that emerge from this sort of calculation. Precision experiments and improved calculations appear to endorse these dreams and ideas, in their supersymmetric version.

Unless this is a cruel tease on the part of Mother Nature, it means we can look forward to a lot of fun exploring supersymmetry, and maybe some aspects of unification, at the LHC. An especially poetic possibility is to explore the possibility that other sorts of parameters, besides gauge couplings, derive by running from a unified value [12. It is widely speculated that the masses of different sorts of gauginos, or of squarks and sleptons, might be related in this way.

\section{From Dark Momentum to Gluonization to Higgs and Dark Matter}

Feynman interpreted the famous SLAC experiments on deep inelastic scattering using an intuitive model of nucleons that postulated point-like particles (partons) as nucleon constituents and treated their dynamics in a crude impulse approximation, ignoring both interactions and quantum interference [13. Identifying the partons as quarks, and building the weak and electromagnetic currents by minimal coupling to quarks, led to many successful predictions 14. There was, however, one clear failure. The momentum carried by quarks inside a fast-moving proton does not add up to the total momentum of the proton, in fact it is less than half.

Today's "dark matter" problem in astronomy is reminiscent of this old "dark momen- 
tum" problem. In the formal treatments of deep inelastic scattering, the analogy becomes eerily precise. In that framework, the (failed) sum rule expresses the equality of the full energy-momentum tensor with the energy-momentum tensor constructed from quarks [5, 6]. Where electroweak currents see just quarks, gravitons see more! We realized early on [5. 6] that the color gluons of QCD, which are electroweak singlets but do carry energymomentum, would enable us to keep the good predictions while losing the bad one. Evidently the gluons had to be major, though "dark" (or better: invisible), constituents of the proton.

Our analysis of deep inelastic scattering, which followed pioneering ideas of Wilson [15], and built on the insightful hard work of Christ, Hasslacher, and Mueller [16, went beyond the parton model in other, more profound ways. A fast-moving quark is revealed, to probes at higher resolution (higher $Q^{2}$ ), to be composed of slower-moving (smaller $x$ ) quarks, antiquarks and gluons, which in turn will resolve into more, softer stuff. This process, seen experimentally as evolution of structure functions, is deeply characteristic of quantum field theory.

These evolution effects further enhance the role of glue in the proton. Several of us worked out that there should be a dramatic pile-up of soft stuff, particularly soft glue, at small $x$ [17. To a hard current (indirectly), or to a hard graviton (theoretically), the proton mostly looks like a blob of soft glue. Twenty years later, beautiful work at HERA confirmed these predictions in impressive detail [18].

Very soft or "wee" constituents of protons played a major role in Feynman's ideas about diffractive scattering [19. His idea was that in diffractive scattering, by exchange of wee partons, the relative phases between different multiparton configurations in the proton wave function get disrupted, without much transfer of energy-momentum. These ideas are intuitively appealing, and have inspired some successful phenomenology, but as far as I know they haven't yet been firmly rooted in QCD.

Much better understood - I hope! - is the importance of gluonization for some frontier topics in high-energy physics, namely Higgs particle production and WIMP searches. The primary, classical coupling of Higgs particles is to quarks, proportional to their mass. But because the $u$ and $d$ quarks we mainly find inside nucleons are so light, their direct coupling is heavily suppressed. Instead the most important coupling arises indirectly, as a quantum effect, through virtual top quark loops connecting to two gluons 20].

I was originally interested in this Higgs-gluon vertex for its potential to induce Higgs particle decays. Georgi, Glashow, Machacek, and Nanopoulos [21] quickly realized it could be exploited for production of Higgs particles, at hadron colliders, through "gluon fusion". This process, which of course relies completely on the glue content of protons, is expected to be the main production mechanism for Higgs particles at the LHC. It is important to calculate the production rate accurately, including good estimates of the gluon distribution functions, so that we will be able to interpret the observed production rate, and check whether the basic vertex is in fact what the standard model, in this intricate way, predicts.

The on-shell Higgs particle couples to hard gluons. In its decay we will see jets, and we can estimate the production using gluon structure functions and perturbative QCD. When considering detection of the sorts of dark-matter candidates provided by models of low-energy supersymmetry we find ourselves involved in quite a different kinematic domain. Since these WIMPs will be heavy and slowly moving by particle physics standards, they 
will scatter at very small momentum transfer. The coupling of SUSY WIMPs to depends on poorly constrained details of the models, but in many realizations it is dominated by virtual Higgs exchange. Here the Higgs-gluon vertex comes in at essentially zero energymomentum. Shifman, Vainshtein and Zakharov [22, in beautiful work, related the relevant gluon operator to the trace of the energy-momentum tensor, whose matrix elements are of course known. This links back to the old dark momentum problem, bringing us full circle.

It is philosophically profound, and quite characteristic of modern physics, that even when viewing something so basic and tangible as a proton, what you see depends very much on how you choose to look. Low-energy electrons see point-like particles, the version described in old high-school textbooks; hard currents see an evolving pattern of quarks; gravitons see these plus lots of gluons as well; wee gluons see some complicated stuff we don't properly understand (we do know its name, Pomeron); real Higgs particles see gluons almost exclusively; and WIMPS, through exchange of virtual Higgs particles, see the Origin of Mass. (The trace of the energy momentum tensor, to which they mainly couple, is on the one hand dominated by contributions from massless color gluons and nearly massless quarks, and on the other hand equal to the nucleon mass.) Each probe reveals different aspects of versatile reality.

\section{From Asymptotic Simplicity to Quark-Gluon Plasma to Quark-Hadron Continuity}

I mentioned earlier how we've learned to use the concept of asymptotic freedom more boldly and confidently over the years. To put it differently, we've learned fruitful ways to lower our standards. Instead of trying to prove directly from first principles that weak coupling applies, we usually content ourselves with consistency checks. That is, we tentatively assume that perturbative calculation of some quantity of interest starting with quark and gluon degrees of freedom is adequate, and check whether the calculation contains infrared divergences [23. This check is by no means trivial, since QCD is full of massless (color) charged particles. So in cases where we find there are no infrared divergences we declare a well-earned victory, and anticipate that our calculations will approximate reality. This strategic retreat has licensed a host of successful applications to describe jet processes, inclusive production, fragmentation, heavy quark physics, and more.

We aren't always forced to compromise. In some important applications, including low-energy spectroscopy, direct integration of the equations using the techniques of lattice gauge theory is practical. But as physicists hungry for answers, we properly regard strict mathematical rigor as a desirable luxury, not an indispensable necessity.

A particularly interesting and important application of the looser philosophy is to construct self-consistent descriptions of extreme states of matter, starting from quarks and gluons 24].

The high temperature, low baryon number regime is foundational for very early universe cosmology. It is also the object of an intense, international experimental program in relativistic heavy ion physics. The overarching theme is that a perturbative description of high-temperature matter, starting with free quarks and gluons, becomes increasingly accurate as the temperature increases. This can be seen, for the equation of state, from nu- 
merical simulation of the full theory [25]. After heroic calculations, which introduce several ingenious new techniques, controlled perturbative calculations (including terms up to sixth order in the coupling, and some infinite resummations) match the numerical work [26. This is a milestone achievement in itself, and also promising for future developments, since the perturbative techniques are more flexible. They might be applied, for example, to calculate viscosity and energy loss, which can be probed experimentally. In this way, we can hope to do justice to the vision of quark-gluon plasma.

The regime of high baryon number density, and low temperature, is intrinsically fascinating, and might be important for describing the inner dynamics of supernovae and the deep interior of neutron stars. The first fundamental result about QCD at high baryon number density is that many of its key properties, including for example the symmetry of the ground state and the energy and charge of the elementary excitations, can not be calculated to a good approximation starting from fermi balls of non-interacting quarks. The perturbation theory (for just about anything) contains infrared divergences [24].

Fortunately, the main source of these divergences is well understood. It signals an instability toward the development of a condensate of quark pairs, similar to the Cooper pairs that occur in metallic superconductors. Whereas the phenomenon of superconductivity in metals is very delicate, because one must overcome the dominant Coulomb repulsion of like charges, color superconductivity is very robust, because there is a fundamentally attractive force between quarks (in the color and flavor antitriplet, spin singlet channel). One can construct an approximate ground state that accommodates the pairs, using the methods of BCS theory. Perturbation theory around this new ground state no longer has infrared divergences. Thus we find that strongly interacting matter at asymptotically high density can be studied using weak coupling, but non-perturbative methods.

Color superconductivity has become an extremely active area of research over the past few years, and many surprises have emerged. Perhaps the most striking and beautiful result is the occurrence of color-flavor locking, a new form of symmetry breaking, in real-world (3 flavor) QCD at asymptotic densities [27. The symmetry $S U(3)_{C} \times S U(3)_{L} \times S U(3)_{R}$ of local color times chiral flavor is broken down to the diagonal subgroup, a residual global $S U(3)$.

Color-flavor locking is a rigorous, calculable consequence of QCD at high density. It implies confinement and chiral symmetry breaking. The low-energy excitations are those created by the quark fields, those created by the gluon fields, and the collective modes associated with chiral symmetry breaking. Because CFL ordering mixes up color and flavor, the quarks form a spin-1/2 octet (plus heavier singlet), the gluons form a vector octet, and the collective modes form a pseudoscalar octet under the residual $S U(3)$. Altogether there is an uncanny resemblance between the properties of dense hadronic matter one calculates for the CFL phase, and the properties one might anticipate for "nuclear matter" in a world with three massless quarks. A nice perspective on this arises if we consider coupling in the $\mathrm{U}(1)$ of electromagnetism. Both the original color gauge symmetry and the original electromagnetic gauge symmetry are broken, but a combination survives. This is similar to what happens in the standard electroweak model, where both weak isospin and hypercharge are broken, but a certain combination survives (to provide electromagnetism). Just as in that case, also in CFL+QED the charge spectrum is modified. One finds that the quarks, gluons, and pseudoscalars acquire integral charges (in units of the electron charge); in fact, 
the charges precisely match those of the corresponding hadrons.

It is difficult to resist the conjecture that these two states are continuously related to one another, with no phase transition, as the density varies [28]. During this variation, degrees of freedom that are "obviously" three-quark baryons evolve continuously into degrees of freedom that are "obviously" single quarks. This nifty trick is possible because diquarks can be exchanged freely with the condensate.

If the core of a neutron star is described by the color-flavor locked (CFL) phase, which seems plausible, it will be a transparent insulator that partially reflects light - like a diamond! This particular consequence of the CFL phase is unlikely to be observed any time soon, but we are working toward defining indirect signatures in observable neutron star and supernova properties.

Unfortunately, existing numerical methods for calculating the behavior of QCD converge very slowly at high density and low temperature. They are totally impractical, even for the biggest and best modern computers. Developing usable algorithms for this kind of problem is a most important open challenge.

There are other stories linking the past with the future through asymptotic freedom and QCD, including a particularly interesting and potentially important one involving axions. But I'll stop here. Thanks again.

\section{References}

[1] R. Jaffe and F. Wilczek, this volume.

[2] D. Gross and F. Wilczek, Phys. Rev. Lett. 30 1343-1346 (1973).

[3] H. Politzer, Phys. Rev. Lett. 30 1346-1349 (1973).

[4] D. Gross and F. Wilczek, Phys. Rev. D8 3633-3652 (1973).

[5] D. Gross and F. Wilczek, Phys. Rev. D9 980-993 (1974).

[6] H. Georgi and H. Politzer, Phys. Rev. D9 416-420 (1974).

[7] N. Nielsen, Am. J. Phys. 49, 1171 (1981); R. Hughes, Nucl. Phys. B186, 376 (1981).

[8] H. Georgi, H. Quinn, and S. Weinberg, Phys. Rev. Lett. 33451 (1974).

[9] J. Pati and A. Salam, Phys. Rev. D8, 1240 (1973).

[10] H. Georgi and S. Glashow, Phys. Rev. Lett. 32, 438 (1974).

[11] S. Dimopoulos, A. Raby, and F. Wilczek, Phys. Rev. D24 1681 (1981).

[12] Reviewed in S. Martin hep-ph/9709356 (see especially his Ref. 75).

[13] R. Feynman, p. 773 in The Past Decade in Particle Theory, ed. E. Sudarshan, Y. Ne'eman (1970). 
[14] J. Bjorken and E. Paschos, Phys. Rev. 185, 1975 (1969).

[15] K. Wilson, Phys. Rev. 179, 1499 (1969); Phys. Rev. D3, 1818 (1971).

[16] N. Christ, B. Hasslacher, and A. Mueller, Phys. Rev. D6 3543 (1972).

[17] A. de Rujula, S. Glashow, H. Politzer, S. Treiman, F. Wilczek, and A. Zee, Phys. Rev. D10 1649 (1974).

[18] Reviewed in G. Wolf, hep-ex/0105055.

[19] R. Feynman, Phys. Rev. Lett. 23, 1415 (1969).

[20] F. Wilczek, Phys. Rev. Lett. 39, 1304 (1977).

[21] H. Georgi, S. Glashow, M. Machacek, and D. Nanopoulos, Phys Rev. Lett. 40, 692 (1978).

[22] M. Shifman, A. Vainshtein, and V. Zakharov, Phys. Lett. B78, 443 (1978).

[23] G. Sterman and S. Weinberg, Phys. Rev. Lett. 39, 1436 (1977).

[24] Reviewed by K. Rajagopal and F. Wilczek, p. 2061 in Handbook of QCD, ed. M. Shifman (2001).

[25] S. Katz, hep-lat/0310051, and references therein.

[26] Y. Schroder and A. Vuorinen, hep-ph/0311323, and references therein.

[27] M. Alford, K. Rajagopal, and F. Wilczek, Nucl. Phys. B537, 443 (1999).

[28] T. Schaefer and F. Wilczek, Phys. Rev. Lett. 82, 3956 (1999). 